\title{
Control of the Fractional-Order Chen Chaotic System via Fractional-Order Scalar Controller and Its Circuit Implementation
}

\author{
Qiong Huang, Chunyang Dong, and Qianbin Chen \\ Key Lab of Mobile Communication Technology, Chongqing University of Posts and Telecommunications, Chongqing 400065, China \\ Correspondence should be addressed to Qianbin Chen; 18502321237@163.com
}

Received 20 March 2014; Revised 26 May 2014; Accepted 4 June 2014; Published 22 June 2014

Academic Editor: Chuandong Li

Copyright (c) 2014 Qiong Huang et al. This is an open access article distributed under the Creative Commons Attribution License, which permits unrestricted use, distribution, and reproduction in any medium, provided the original work is properly cited.

\begin{abstract}
A fractional-order scalar controller which involves only one state variable is proposed. By this fractional-order scalar controller, the unstable equilibrium points in the fractional-order Chen chaotic system can be asymptotically stable. The present control strategy is theoretically rigorous. Some circuits are designed to realize these control schemes. The outputs of circuit agree with the results of theoretical results.
\end{abstract}

\section{Introduction}

In the last few decades, chaotic behaviors have been discovered in many areas of science and engineering such as mathematics, physics, chemistry, electronics, medicine, economy, biological science, and social science. In 1990, Ott et al. presented the OGY method of chaotic control [1]. After that, chaos control has attracted increasing attention among scientists in various fields. Many control schemes $[1,2]$ have been presented, such as feedback control, parametric perturbation control, adaptive control, and fuzzy control. On the other hand, the chaotic or hyperchaos behaviors have been found in many fractional-order dynamical systems. Many fractional-order chaotic systems have been presented, the fractional-order Chua's chaotic circuit [3], the fractional-order Duffing chaotic system [4], the fractionalorder memristor-based chaotic system [5], the fractionalorder Lorenz chaotic system [6], the fractional-order Chen chaotic system [7], and so forth [8,9]. Moreover, control and synchronization of fractional-order chaotic systems have attracted much attention in the recent years [10-16].

Compared to the traditional controller (integer-order controller), the fractional-order controller has many advantages, such as less sensitivity to parameter variations and better disturbance rejection ratios [17]. It is possible that traditional controller (integer-order controller) will be replaced by fractional-order controller in the future. Recently, a fractional-order vector controller is addressed to stabilize the unstable equilibrium points for integer-order chaotic systems by Tavazoei and Haeri [17]. Zhou and Kuang have presented another fractional-order vector controller to stabilize the nonequilibrium points for integer-order chaotic systems [18]. However, only integer-order chaotic systems are discussed in $[17,18]$, and only fractional-order vector controller is investigated.

Up to now, to the best of our knowledge, very few results on chaotic control are reported by fractional-order scalar controller. Motivated by the above-mentioned discussions, some fractional-order scalar controllers are presented to control the fractional-order Chen chaotic systems in this paper. Only one system state variable is used in the fractionalorder scalar controller. The control scheme is simple and theoretical. Moreover, some circuits are designed to realize these control schemes, and the circuit results agree with the theoretical results.

The outline of this paper is as follows. In Section 2, some mathematical preliminaries are addressed for the fractionalorder system. In Section 3, some fractional-order scalar controller are proposed to stabilize the unstable equilibrium points in the fractional-order Chen chaotic system. In Section 4, some circuits are designed to realize the control schemes. The conclusion is finally drawn in Section 5. 


\section{Mathematical Preliminaries}

In this paper, we use the Caputo definition of fractional derivative, which is

$$
D^{q} h(t)=\frac{1}{\Gamma(l-q)} \int_{0}^{t} h^{(l)}(\tau)(t-\tau)^{l-q-1} d \tau, \quad l-1<q<l,
$$

where $D^{q}$ denoted the Caputo operator, $l$ is the first integer which is not less than $q$, and $h^{(l)}(t)$ is the $l$-order derivative for $h(t)$; that is, $h^{(l)}(t)=d^{l} h(t) / d t^{l}$.

Consider the following nonlinear fractional-order system:

$$
D^{q} x=F(x)
$$

where $F: R^{n} \rightarrow R^{n}$ are continuous function, $0<q<1$ are fractional order, and $x \in R^{n}$ are state vectors.

First, we recall the stability results of nonlinear fractionalorder systems [19-24]. Let the equilibrium point of system (2) be $x_{0}$ and let the Jacobian matrix be $\partial F /\left.\partial x\right|_{x=x_{0}}$. $\lambda_{i}(i=1,2, \ldots, n)$ are the eigenvalues of the Jacobian matrix $\partial F /\left.\partial x\right|_{x=x_{0}}$. If $\left|\arg \lambda_{i}\right|>0.5 \pi q(i=1,2, \ldots, n)$ are satisfied, then the equilibrium point $x_{0}$ is asymptotically stable [19-24].

Second, we recall the improved version of AdamsBashforth-Moulton algorithm [14] for the fractional-order systems. Consider the following two-dimensional nonlinear fractional-order system:

$$
\begin{aligned}
& D^{q_{1}} x_{1}=h_{1}\left(x_{1}, x_{2}\right), \\
& D^{q_{2}} x_{2}=h_{2}\left(x_{1}, x_{2}\right),
\end{aligned}
$$

with initial condition $\left(h_{1}(0), h_{2}(0)\right)$. Let $\tau=T / N$ and let $t_{n}=$ $n \tau(n=0,1,2, \ldots, N)$. Then, the two-dimensional fractionalorder system can be discretized as follows

$$
\begin{aligned}
& x_{1}(n+1) \\
& \left.\begin{array}{rl}
=h_{1}(0)+\frac{\tau^{q_{1}}}{\Gamma\left(q_{1}+2\right)}[ & h_{1}\left(x_{1}^{m}(n+1), x_{2}^{m}(n+1)\right) \\
& \left.+\sum_{j=0}^{n} \kappa_{1, j, n+1} h_{1}\left(x_{1}(j), x_{2}(j)\right)\right], \\
x_{2}(n+1) & {\left[\begin{array}{c}
\tau^{q_{2}} \\
h_{2}\left(x_{1}^{m}(n+1), x_{2}^{m}(n+1)\right)
\end{array}\right.} \\
+h_{2}(0)+\kappa_{2, j, n+1} h_{2}\left(x_{1}(j), x_{2}(j)\right)
\end{array}\right],
\end{aligned}
$$

where

$$
\begin{gathered}
x_{1}^{m}(n+1) \\
=x_{1}(0)+\frac{1}{\Gamma\left(q_{1}\right)} \sum_{j=0}^{n} \sigma_{1, j, n+1} h_{1}\left(x_{1}(j), x_{2}(j)\right), \\
x_{2}^{m}(n+1) \\
=x_{2}(0)+\frac{1}{\Gamma\left(q_{2}\right)} \sum_{j=0}^{n} \sigma_{2, j, n+1} h_{2}\left(x_{1}(j), x_{2}(j)\right), \\
\kappa_{i, j, n+1} \quad \begin{array}{ll}
n^{q_{i}+1}-\left(n-q_{i}\right)(n+1)^{q_{i}}, & j=0, \\
(n-j+2)^{q_{i}+1}+(n-j)^{q_{i}+1} & \\
-2(n-j+1)^{q_{i}+1}, & j=n+1, \quad(i=1,2), \\
1, &
\end{array} \\
\sigma_{i, j, n+1}=\frac{\tau^{q_{i}}}{q_{i}}\left[(n-j+1)^{q_{i}}-(n-j)^{q_{i}}\right], \\
0 \leq j \leq n, \quad(i=1,2) .
\end{gathered}
$$

The error of this algorithm is

$$
\begin{gathered}
\left|x_{i}\left(t_{n}\right)-x_{i}(n)\right|=o\left(\tau^{\alpha_{i}}\right), \\
\alpha_{i}=\min \left(2,1+q_{i}\right), \quad(i=1,2) .
\end{gathered}
$$

\section{Control of the Unstable Equilibrium Points for the Fractional-Order Chen Chaotic System via a Fractional-Order Scalar Controller}

In this section, some fractional-order scalar controllers which involve only one state variable are addressed. The unstable equilibrium points of the fractional-order Chen chaotic system can be asymptotically stable by these fractional-order scalar controllers.

In 1963, E. N. Lorenz reported the first chaotic model that revealed the complex and fundamental behaviors of the nonlinear dynamical systems. In 1999, Chen found another chaotic model in a simple three-dimensional autonomous system, which nevertheless is not topologically equivalent to the Lorenz chaotic model. The fractional-order Chen chaotic model is described as

$$
\begin{gathered}
D^{q} x_{1}=35\left(x_{2}-x_{1}\right), \\
D^{q} x_{2}=-7 x_{1}+28 x_{2}-x_{1} x_{3}, \\
D^{q} x_{3}=x_{1} x_{2}-3 x_{3},
\end{gathered}
$$

where $0<q<1$ is the fractional order. The fractional-order Chen chaotic system has chaotic attractor for $q \geq 0.83$ [19]. The fractional-order Chen chaotic attractor with $q=0.9$ is shown as in Figure 1. 


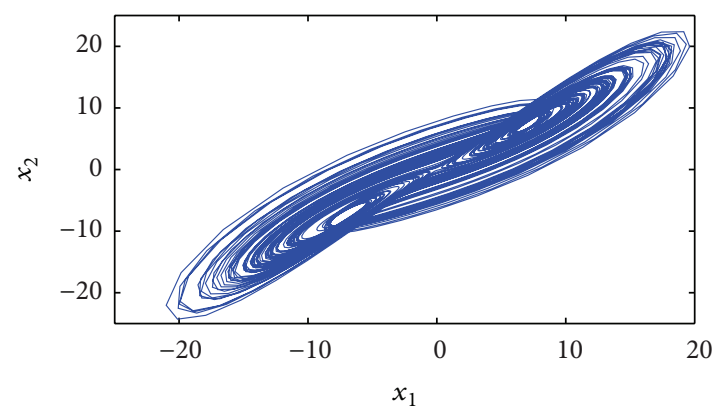

(a)

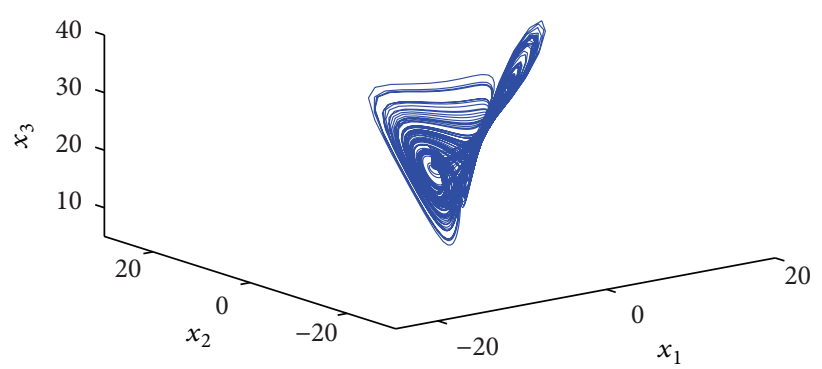

(b)

Figure 1: The fractional-order Chen chaotic attractor with $q=0.9$.

There are three unstable equilibrium points in the above fractional-order Chen chaotic system. The unstable equilibrium points are $S_{0}=(0,0,0)$ and $S_{ \pm}=( \pm \sqrt{63}, \pm \sqrt{63}, 21)$, respectively. Our goal is how to control the unstable equilibrium points via a fractional-order scalar controller.

3.1. Case 1: Control of the Unstable Equilibrium Point $S_{0}=$ $(0,0,0)$

Theorem 1. Let the controlled system be

$$
\begin{gathered}
D^{q} x_{1}=35\left(x_{2}-x_{1}\right)+l_{1} D^{q} x_{2}+l_{2} x_{2}, \\
D^{q} x_{2}=-7 x_{1}+28 x_{2}-x_{1} x_{3}, \\
D^{q} x_{3}=x_{1} x_{2}-3 x_{3},
\end{gathered}
$$

where $l_{1} D^{q} x_{2}+l_{2} x_{2}$ is the scalar fractional-order controller and $l_{1}$ and $l_{2}$ are feedback coefficients. If $l_{1}>-1$ and $l_{2}>105$, then the controlled system (8) will be asymptotically converged to the equilibrium point $S_{0}=(0,0,0)$.

Proof. The unstable equilibrium point $S_{0}=(0,0,0)$ in the fractional-order Chen chaotic system is also the equilibrium point in the controlled system (8). The Jacobi matrix of the controlled system at equilibrium point $S_{0}=(0,0,0)$ is

$$
J_{(0,0,0)}=\left|\begin{array}{ccc}
-35-7 l_{1} & 35+28 l_{1}+l_{2} & 0 \\
-7 & 28 & 0 \\
0 & 0 & -3
\end{array}\right| .
$$

The eigenvalues are

$$
\begin{aligned}
\lambda_{ \pm}= & -0.5\left(7+7 l_{1}\right) \\
& \pm 0.5 \sqrt{\left(7+7 l_{1}\right)^{2}-4\left(7 l_{2}-21 \times 35\right)}, \quad \lambda_{3}=-3,
\end{aligned}
$$

because

$$
l_{1}>-1, \quad l_{2}>105 .
$$

So

$$
\operatorname{Re}\left(\lambda_{ \pm}\right)<0 .
$$

Therefore, all eigenvalues of the Jacobi matrix at equilibrium point $S_{0}=(0,0,0)$ in the controlled system ( 8$)$ have negative real part. This result implies that the controlled system will be asymptotically converged to the equilibrium point $S_{0}=$ $(0,0,0)$. The proof is completed.

Theorem 2. Consider the controlled system is as follows:

$$
\begin{gathered}
D^{q} x_{1}=35\left(x_{2}-x_{1}\right), \\
D^{q} x_{2}=-7 x_{1}+28 x_{2}-x_{1} x_{3}+l_{3} D^{q} x_{1}+l_{4} x_{1}, \\
D^{q} x_{3}=x_{1} x_{2}-3 x_{3},
\end{gathered}
$$

where $l_{3} D^{q} x_{2}+l_{4} x_{2}$ is a fractional-order scalar controller and $l_{3}$ and $l_{4}$ are feedback coefficients. If $l_{3}<0.2$ and $-\left(35 l_{3}-7\right)^{2} / 140 \leq l_{4}+21<0$, then the controlled system (13) will be asymptotically converged to the equilibrium point $S_{0}=(0,0,0)$.

Proof. It is easily to obtain that the unstable equilibrium point $S_{0}=(0,0,0)$ in the fractional-order Chen chaotic system is also the equilibrium point in the controlled system (13). The Jacobi matrix of the controlled system (13) at equilibrium point $S_{0}=(0,0,0)$ is

$$
J_{(0,0,0)}=\left|\begin{array}{ccc}
-35 & 35 & 0 \\
-7-35 l_{3}+l_{4} & 28+35 l_{3} & 0 \\
0 & 0 & -3
\end{array}\right| .
$$

The eigenvalues are

$$
\begin{aligned}
\lambda_{ \pm}= & 0.5\left(35 l_{3}-7\right) \\
& \pm 0.5 \sqrt{\left(35 l_{3}-7\right)^{2}+140\left(21+l_{4}\right)}, \quad \lambda_{3}=-3,
\end{aligned}
$$

because

$$
l_{3}<0.2, \quad-\frac{\left(35 l_{1}-7\right)^{2}}{140} \leq l_{4}+21<0 .
$$

So

$$
\operatorname{Re}\left(\lambda_{ \pm}\right)<0 .
$$


Therefore, all eigenvalues of the Jacobi matrix at equilibrium point $S_{0}=(0,0,0)$ in the controlled system (13) have negative real part. This result indicates that the controlled system (13) will be asymptotically converged to the equilibrium point $S_{0}=(0,0,0)$. The proof is completed.

\subsection{Case 2: Control of the Unstable Equilibrium Points $S_{+}=$} $( \pm \sqrt{63}, \pm \sqrt{63}, 21)$

Theorem 3. Consider the controlled system is

$$
\begin{gathered}
D^{q} x_{1}=35\left(x_{2}-x_{1}\right), \\
D^{q} x_{2}=-7 x_{1}+28 x_{2}-x_{1} x_{3}+l_{5} D^{q} x_{1}, \\
D^{q} x_{3}=x_{1} x_{2}-3 x_{3},
\end{gathered}
$$

where $l_{5} D^{q} x_{1}$ is the scalar fractional-order controller and $l_{5}$ is feedback coefficient. If $35 l_{5}<19-\sqrt{1551}$, then the controlled system (18) will be asymptotically converged to the equilibrium point $S_{+}=(\sqrt{63}, \sqrt{63}, 21)$.

Proof. The Jacobian matrix at the equilibrium point $S_{+}=$ $(\sqrt{63}, \sqrt{63}, 21)$ in the controlled system $(18)$ is

$$
J=\left|\begin{array}{ccc}
-35 & 35 & 0 \\
-28-35 l_{5} & 28+35 l_{5} & -\sqrt{63} \\
\sqrt{63} & \sqrt{63} & -3
\end{array}\right| .
$$

Its characteristic equation is

$$
\lambda^{3}+c_{1} \lambda^{2}+c_{2} \lambda+c_{3}=0
$$

where $c_{1}=10-35 l_{5}, c_{2}=3\left(28-35 l_{5}\right)$, and $c_{3}=4410$.

Because $35 l_{5}<19-\sqrt{1551}$, the following yields

$$
c_{1}>0, \quad c_{2}>0, \quad c_{1} c_{2}-c_{3}>0
$$

This result indicates that all eigenvalues of the Jacobi matrix at equilibrium point $S_{+}=(\sqrt{63}, \sqrt{63}, 21)$ in the controlled system (18) have negative real part. So, the controlled system (18) will be asymptotically converged to the equilibrium point $S_{+}=(\sqrt{63}, \sqrt{63}, 21)$. The proof is completed.

Similarly, we can easily control the fractional-order Chen chaotic system that will be asymptotically converged to the unstable equilibrium point $S_{-}=(-\sqrt{63},-\sqrt{63}, 21)$.

Remark 4. In this section, we only discuss that all eigenvalues of the Jacobi matrix at equilibrium point in the controlled system have negative real part. Recently, Li and Ma [25] reported the more rigorous result on the local asymptotical stability of the nonlinear fractional differential system. Their result also can be applied to control the unstable equilibrium point in the fractional-order Chen chaotic system.

Remark 5. Only one system state variable and its fractionalorder derivative are used in our fractional-order scalar controller. This is the main contribution in our work.

\section{Circuit Implementation of the Control Scheme for the Fractional-Order Chen Chaotic System}

In this subsection, some circuits are designed to realize these control schemes for the fractional-order Chen chaotic system, and the circuit results fit the theoretical results mentioned in Section 3.

Now, many references on the guidelines to design circuits for the fractional-order chaotic systems are reported. By the circuit design methods [9, 26-29], the circuits are designed as mentioned below to realize the fractional-order chaotic system (8), (13), and (18), and the circuit experiments are obtained.

4.1. Case 1: Realize Physically the Controlled Fractional-Order Chen Chaotic System (8). Now, let $l_{1}=1$ and $l_{2}=200$ in the controlled system (8). According to Theorem 1, the controlled system (8) will be asymptotically converged to the unstable equilibrium point $S_{0}=(0,0,0)$. By the circuit design method $[9,27,28]$, the circuit diagram designed to realize the controlled system (8) is presented as shown in Figures 2 and 3.

The first equation, the second equation, and the third equation in controlled system (8) are realized by Figures 2(a), $2(\mathrm{~b})$, and 2(c), respectively. The operator $d^{q} / d t^{q}$ is realized by Figure 3.

According to the circuit design methods, the resistors in Figure 2 are chosen as $R_{1}=100 \mathrm{k} \Omega, R_{2}=2.86 \mathrm{k} \Omega, R_{3}=$ $3.57 \mathrm{k} \Omega, R_{4}=14.3 \mathrm{k} \Omega, R_{5}=33.3 \mathrm{k} \Omega, R_{6}=100 \mathrm{k} \Omega$, and $R_{7}=0.5 \mathrm{k} \Omega$, respectively. Here and later, the capacitors and resistors in Figure 3 are chosen as $R_{11}=62.84 \mathrm{M} \Omega, R_{22}=$ $0.25 \mathrm{M} \Omega, R_{33}=0.0025 \mathrm{M} \Omega, C_{11}=1.232 \mu \mathrm{F}, C_{22}=1.84 \mu \mathrm{F}$, and $C_{33}=1.1 \mu \mathrm{F}$. The operational amplifiers are of the type of LF353N, the multipliers are of the type of AD633, and the power is supplied by $\pm 15 \mathrm{~V}$.

By choosing the circuit output $x_{1}$ in Figure $2(a)$ as the vertical axis input, Figure 4(a) shows the circuit experiment displayed on the oscilloscope. Similarly, Figure 4(b) shows the circuit experiment displayed on the oscilloscope with the circuit outputs $x_{2}$ in Figure 2(b) and Figure 4(c) shows the circuit experiment displayed on the oscilloscope with the circuit outputs $x_{3}$ in Figure 2(c). In this paper, the vertical coordinate unit is $\mathrm{V}$ (volt) and the horizontal coordinate unit is second $(\mathrm{s})$.

According to Figure 4, the circuit results fit the theoretical results mentioned in Theorem 1 .

4.2. Case 2: Realize Physically the Controlled Fractional-Order Chen Chaotic System (13). Now, let $l_{3}=-1$ and $l_{4}=-30$ in the controlled system (13). According to Theorem 2, the controlled system (8) will be asymptotically converged to the unstable equilibrium point $S_{0}=(0,0,0)$. Similarly, the circuit diagram designed to realize the controlled system (13) is as shown in Figure 5.

Here, the first equation, the second equation, and the third equation in controlled system (13) are realized by Figures 5(a), 5(b), and 5(c), respectively. The operator $d^{q} / d t^{q}$ is realized by Figure 3. 


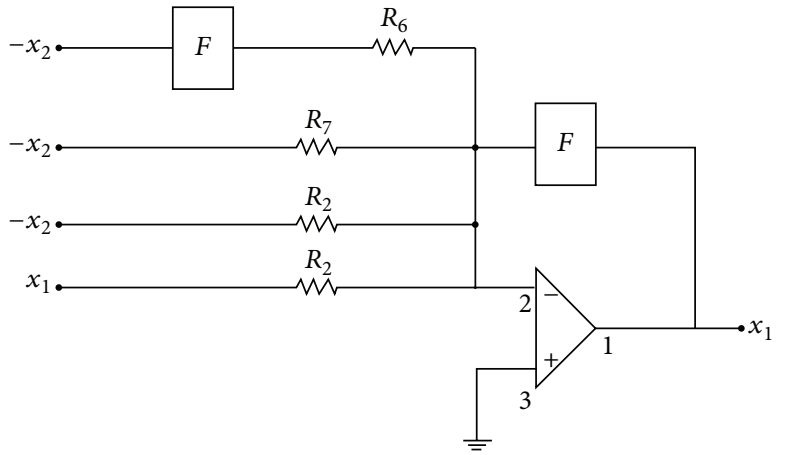

(a)

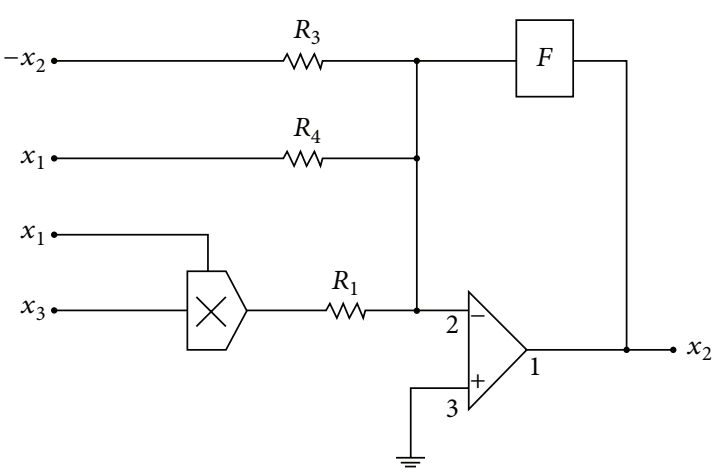

(b)

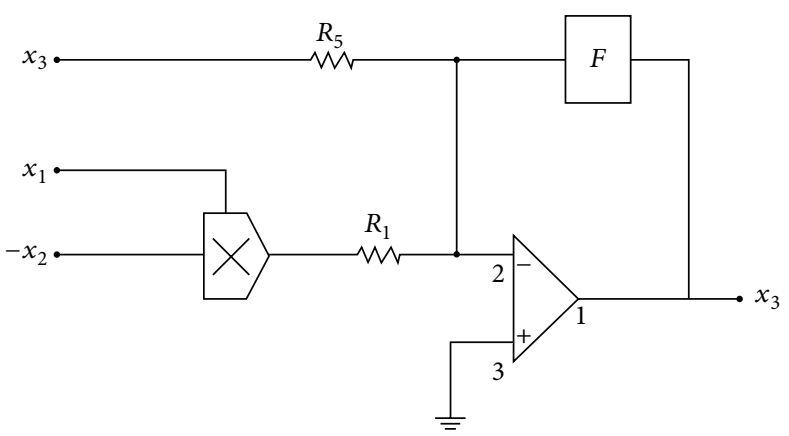

(c)

FIGURE 2: The circuit diagram designed to realize the fractional-order controlled system (8) for $q=0.9$.

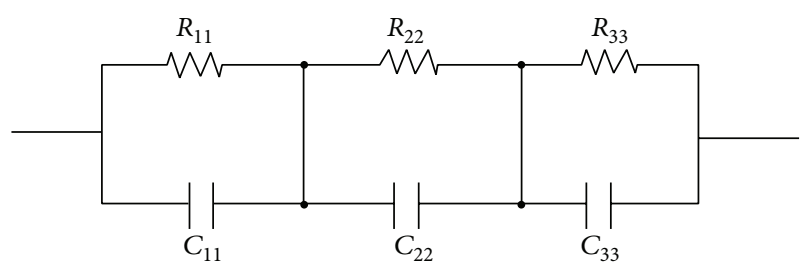

Figure 3: Circuit diagram for box $F$.

According to the circuit design methods, the resistors in Figure 5 are chosen as $R_{8}=100 \mathrm{k} \Omega$, and $R_{9}=3.33 \mathrm{k} \Omega$, respectively. The resistors $R_{i}(i=1,2, \ldots, 7)$ are the same as in Figure 2.

Similarly, by choosing the circuit output $x_{1}$ in Figure 5(a) as the vertical axis input, Figure 6(a) shows the circuit experiment displayed on the oscilloscope. Similarly, Figure 6(b) shows the circuit experiment displayed on the oscilloscope with the circuit outputs $x_{2}$ in Figure 5(b) and Figure 6(c) shows the circuit experiment displayed on the oscilloscope with the circuit outputs $x_{3}$ in Figure 5(c).

According to Figure 6, the circuit results agree with the theoretical results mentioned in Theorem 2.

\subsection{Case 3: Realize Physically the Controlled Fractional-Order} Chen Chaotic System (18). Now, let $l_{5}=-1$ in the controlled system (18). According to Theorem 3, the controlled system (18) will be asymptotically converged to the unstable equilibrium point $S_{+}=(\sqrt{63}, \sqrt{63}, 21)$. Similarly, the circuit diagram designed to realize the controlled system (18) is displayed as shown in Figure 7.

Similarly, the first equation, the second equation, and the third equation in controlled system (18) are realized by Figures 7(a), 7(b), and 7(c), respectively. The operator $d^{q} / d t^{q}$ is realized by Figure 3. The resistors and capacitors in Figure 7 are chosen as Case 1 and Case 2.

By choosing the circuit output $x_{1}$ in Figure $7(a)$ as the vertical axis input, Figure 8(a) shows the circuit experiment displayed on the oscilloscope. Similarly, Figure 8(b) shows the circuit experiment displayed on the oscilloscope with the circuit outputs $x_{2}$ in Figure 7(b) and Figure 8(c) shows the circuit experiment displayed on the oscilloscope with the circuit outputs $x_{3}$ in Figure 7(c).

According to Figure 8, the circuit results agree with the theoretical results mentioned in Theorem 3.

\section{Conclusions}

In order to control of the unstable equilibrium points for the fractional-order Chen chaotic system, some fractionalorder scalar controllers are proposed, and only one state variable is used in the fractional-order scalar controller. The control scheme is theoretically rigorous. Moreover, three fractional-order chaotic circuits are designed to realize the control strategy, and the circuit experiments are obtained. The experiment results agree with the theoretical results. Furthermore, some results [30-33] on the effect of noises or disturbances in control or synchronization problems of chaotic systems have been proposed. The anticontrol or 


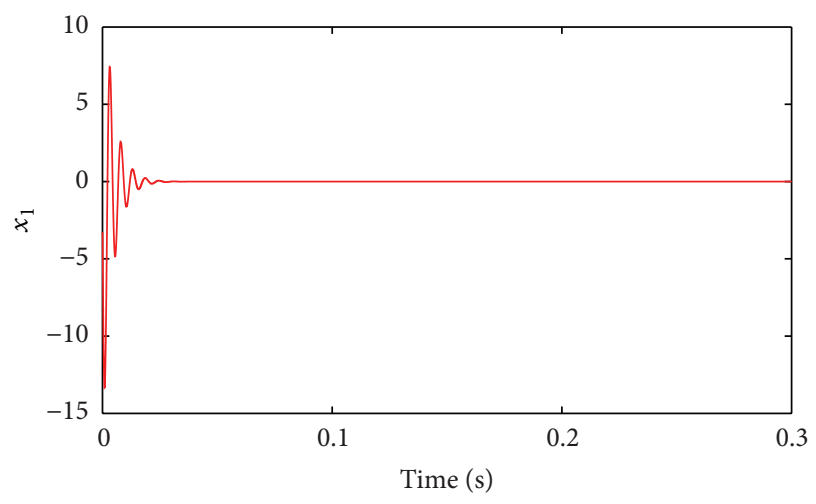

(a)

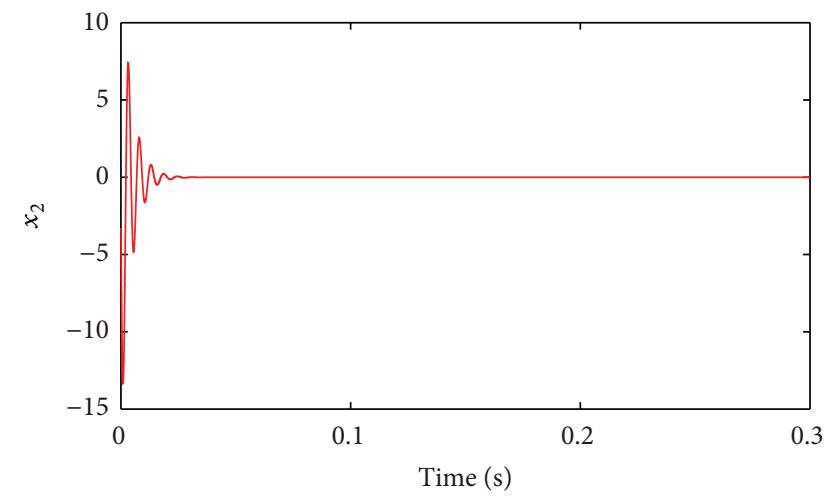

(b)

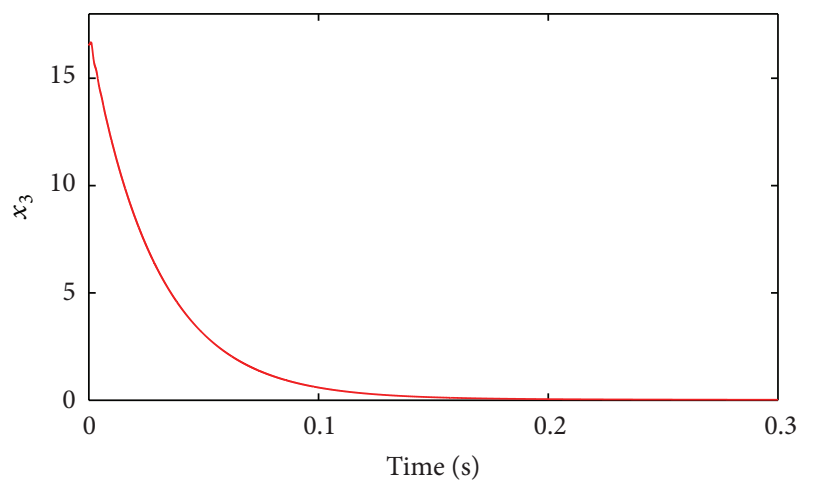

(c)

Figure 4: The circuit experiment displayed on the oscilloscope.

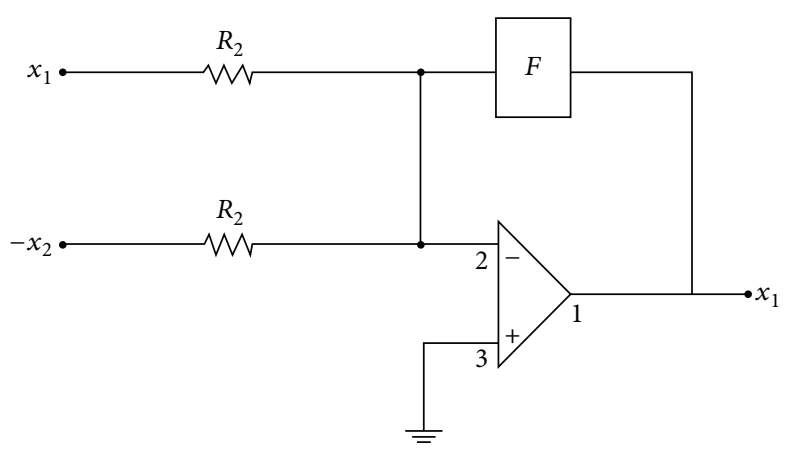

(a)

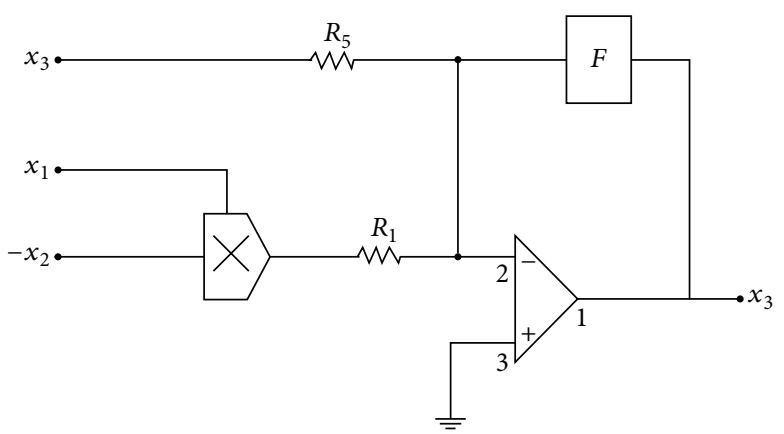

(c)

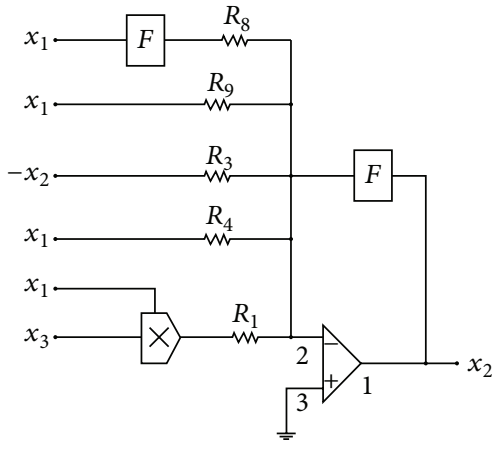

(b) 


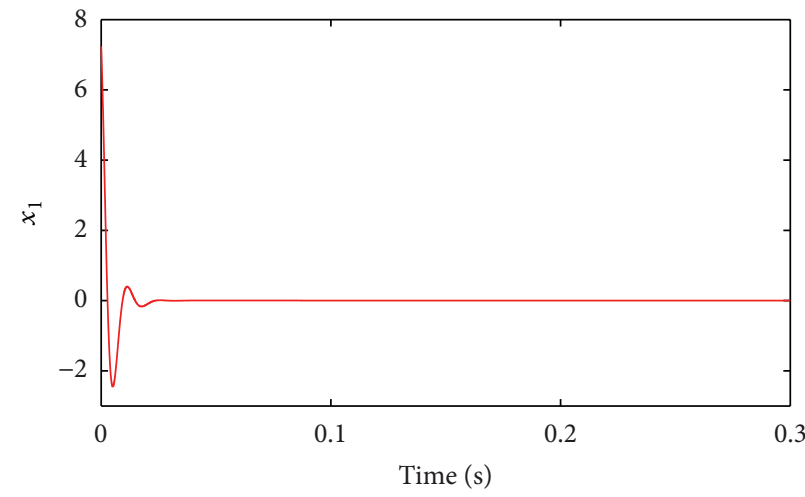

(a)

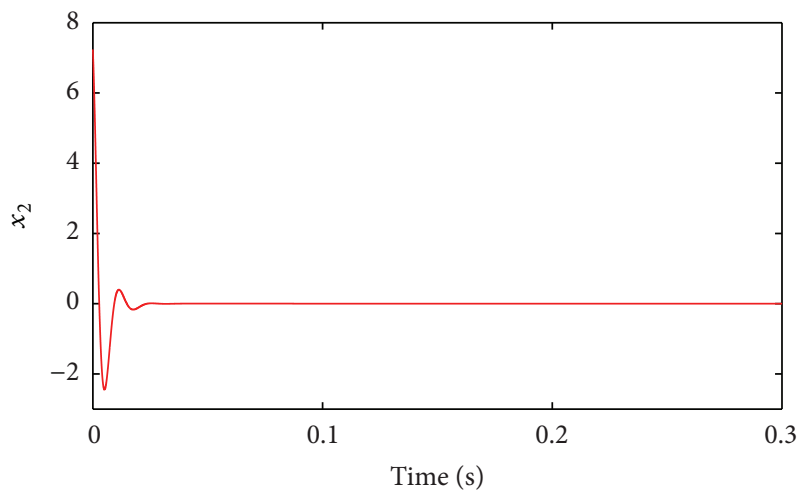

(b)

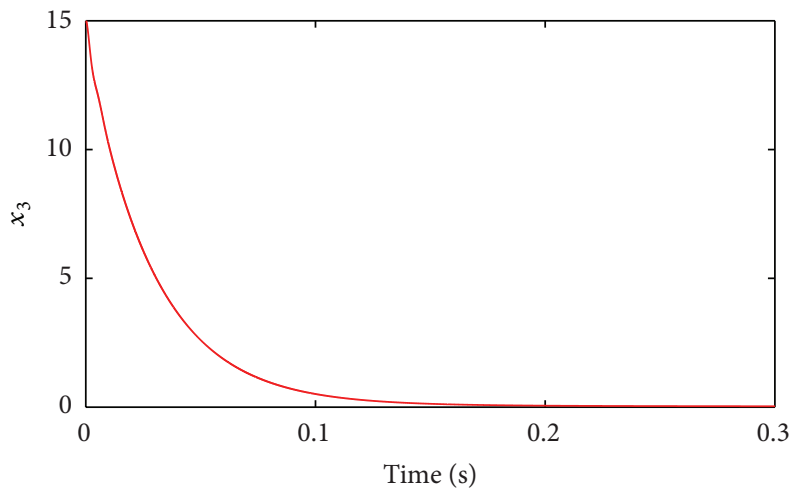

(c)

FIGURE 6: The circuit experiment displayed on the oscilloscope.

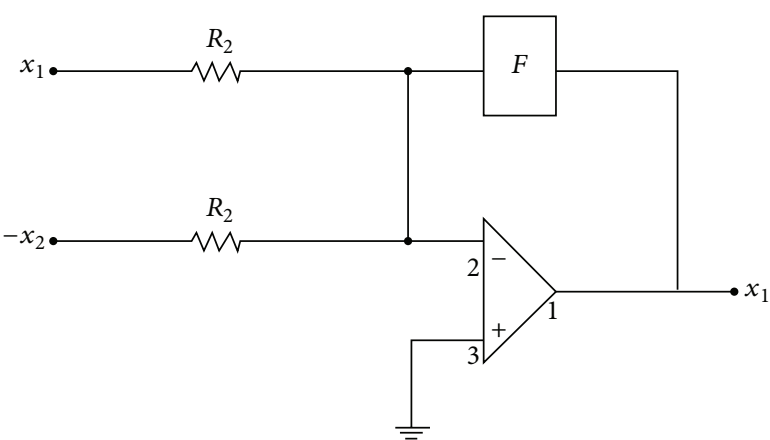

(a)

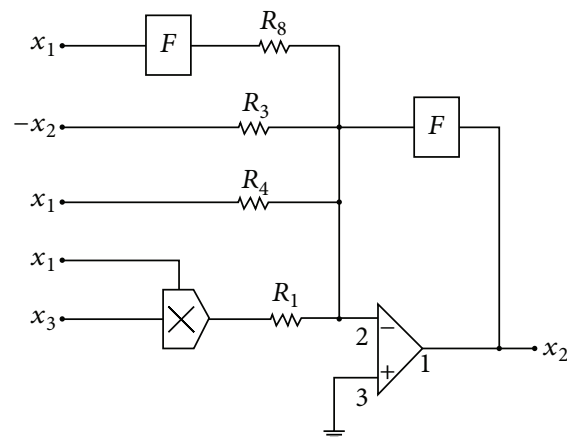

(b)

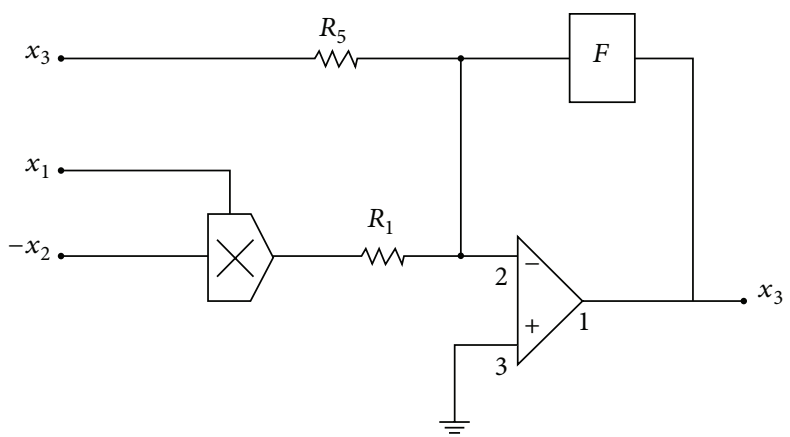

(c)

FIGURE 7: The circuit diagram designed to realize the fractional-order controlled system (18) for $q=0.9$. 


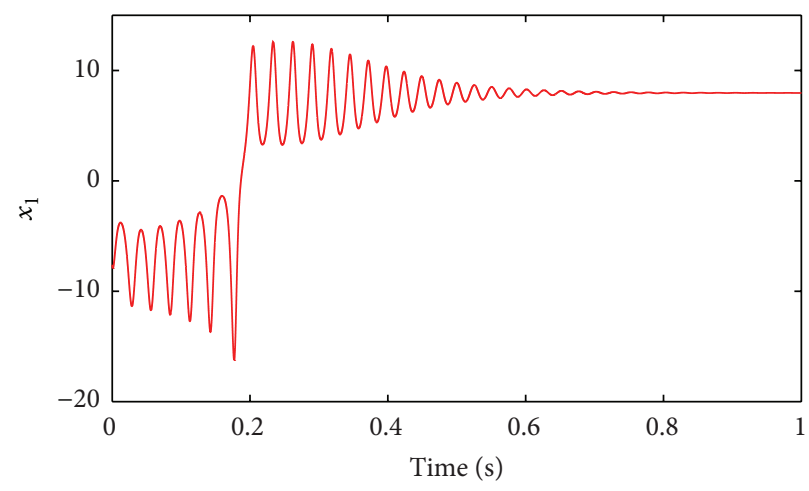

(a)

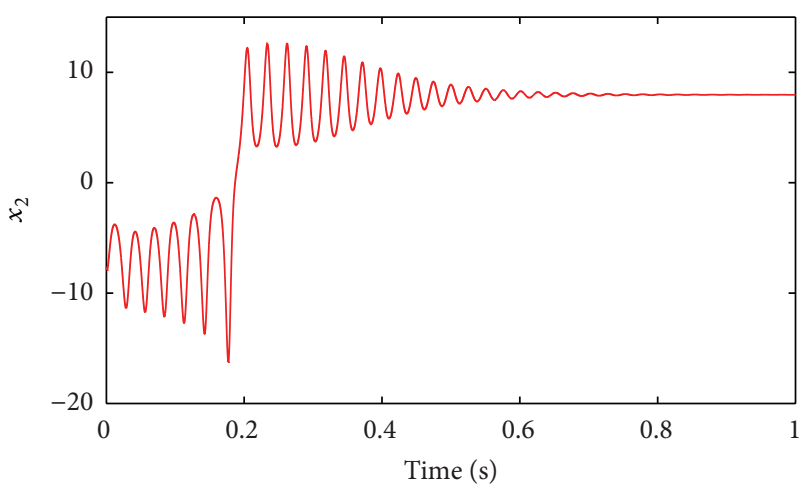

(b)

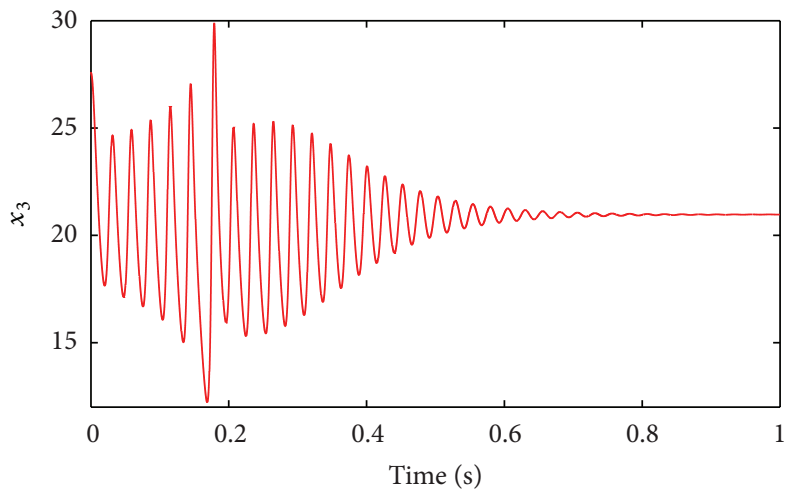

(c)

Figure 8: The circuit experiment displayed on the oscilloscope.

antisynchronization problems for fractional chaotic systems with disturbances or noises have been also discussed in [34]. So, the effect of noises or disturbances for our control scheme is our further work.

\section{Conflict of Interests}

The authors declare that there is no conflict of interests regarding the publication of this paper.

\section{Acknowledgments}

This work is supported by the National Science and Technology Specific Project of China (2012YQ20022404), National Natural Science Foundation of China (no. 60972070), Program for Changjiang Scholars and Innovative Research Team in University (IRT1299), and the special fund of Chongqing key laboratory (CSTC).

\section{References}

[1] E. Ott, C. Grebogi, and J. A. Yorke, "Controlling chaos," Physical Review Letters, vol. 64, no. 11, pp. 1196-1199, 1990.

[2] G. Chen and X. Yu, Chaos Control: Theory and Applications, Springer, Berlin, Germany, 2003.

[3] T. T. Hartley, C. F. Lorenzo, and H. K. Qammer, "Chaos in a fractional order Chua's system," IEEE Transactions on Circuits and Systems I: Fundamental Theory and Applications, vol. 42, no. 8, pp. 485-490, 1995.

[4] Z. M. Ge and C. Y. Ou, "Chaos in a fractional order modified Duffing system," Chaos, Solitons and Fractals, vol. 34, no. 2, pp. 262-291, 2007.

[5] D. Cafagna and G. Grassi, "On the simplest fractional-order memristor-based chaotic system," Nonlinear Dynamics, vol. 70, no. 2, pp. 1185-1197, 2012.

[6] I. Grigorenko and E. Grigorenko, "Chaotic dynamics of the fractional Lorenz system," Physical Review Letters, vol. 91, no. 3, pp. 034101-034104, 2003.

[7] C. Li and G. Peng, "Chaos in Chen's system with a fractional order," Chaos, Solitons and Fractals, vol. 22, no. 2, pp. 443-450, 2004.

[8] P. Zhou and F. Y. Yang, "Hyperchaos, chaos, and horseshoe in a $4 \mathrm{D}$ nonlinear system with an infinite number of equilibrium points," Nonlinear Dynamics, vol. 76, no. 1, pp. 473-480, 2014.

[9] P. Zhou and K. Huang, "A new 4-D non-equilibrium fractionalorder chaotic system and its circuit implementation," Communications in Nonlinear Science and Numerical Simulation, vol. 19, no. 6, pp. 2005-2011, 2014.

[10] P. Zhou, R. Ding, and Y. Cao, "Multi drive-one response synchronization for fractional-order chaotic systems," Nonlinear Dynamics, vol. 70, no. 2, pp. 1263-1271, 2012.

[11] L. Pan, W. Zhou, L. Zhou, and K. Sun, "Chaos synchronization between two different fractional-order hyperchaotic systems," Communications in Nonlinear Science and Numerical Simulation, vol. 16, no. 6, pp. 2628-2640, 2011. 
[12] S. S. Delshad, M. M. Asheghan, and M. H. Beheshti, "Synchronization of $\mathrm{N}$-coupled incommensurate fractional-order chaotic systems with ring connection," Communications in Nonlinear Science and Numerical Simulation, vol. 16, no. 9, pp. 3815-3824, 2011.

[13] H. Taghvafard and G. H. Erjaee, "Phase and anti-phase synchronization of fractional order chaotic systems via active control," Communications in Nonlinear Science and Numerical Simulation, vol. 16, no. 10, pp. 4079-4088, 2011.

[14] R. X. Zhang and S. P. Yang, "Adaptive synchronization of fractional-order chaotic systems via a single driving variable," Nonlinear Dynamics, vol. 66, no. 4, pp. 831-837, 2011.

[15] D. Cafagna and G. Grassi, “Observer-based projective synchronization of fractional systems via a scalar signal: application to hyperchaotic Rössler systems," Nonlinear Dynamics, vol. 68, no. 1-2, pp. 117-128, 2012.

[16] R. Zhang and S. Yang, "Stabilization of fractional-order chaotic system via a single state adaptive-feedback controller," Nonlinear Dynamics, vol. 68, no. 1-2, pp. 45-51, 2012.

[17] M. S. Tavazoei and M. Haeri, "Chaos control via a simple fractional-order controller," Physics Letters A, vol. 372, no. 6, pp. 798-807, 2008.

[18] P. Zhou and F. Kuang, "A novel control method for integer orders chaos systems via fractional-order derivative," Discrete Dynamics in Nature and Society, vol. 2011, Article ID 217843, 8 pages, 2011.

[19] P. Zhou and W. Zhu, "Function projective synchronization for fractional-order chaotic systems," Nonlinear Analysis: Real World Applications, vol. 12, no. 2, pp. 811-816, 2011.

[20] D. Matignon, "Stability results for fractional differential equations with application to control processing," in Proceedings of the International IMACS IEEE-SMC Multiconference on Computational Engineering in Systems Applications, pp. 9631031, Lille, France, July 1996.

[21] E. Ahmed, A. M. A. El-Sayed, and H. A. A. El-Saka, "Equilibrium points, stability and numerical solutions of fractionalorder predator-prey and rabies models," Journal of Mathematical Analysis and Applications, vol. 325, no. 1, pp. 542-553, 2007.

[22] Z. M. Odibat, "Adaptive feedback control and synchronization of non-identical chaotic fractional order systems," Nonlinear Dynamics, vol. 60, no. 4, pp. 479-487, 2010.

[23] Z. Odibat, "A note on phase synchronization in coupled chaotic fractional order systems," Nonlinear Analysis: Real World Applications, vol. 13, no. 2, pp. 779-789, 2012.

[24] X. Wang, Y. He, and M. Wang, "Chaos control of a fractional order modified coupled dynamos system," Nonlinear Analysis: Theory, Methods and Applications, vol. 71, no. 12, pp. 6126-6134, 2009.

[25] C. P. Li and Y. T. Ma, "Fractional dynamical system and its linearization theorem," Nonlinear Dynamics, vol. 71, pp. 621633, 2013.

[26] F. Q. Wang and C. X. Liu, "Study on the critical chaotic system with fractional order and circuit experiment," Acta Physica Sinica, vol. 55, pp. 3922-3927, 2006.

[27] P. Zhou, X. Cheng, and M. Zhang, "One new fractionalorder chaos system and its circuit simulation by electronic workbench," Chinese Physics B, vol. 17, no. 9, pp. 3252-3257, 2008.

[28] H. Q. Li, X. F. Liao, and M. W. Luo, "A novel non-equilibrium fractional-order chaotic system and its complete synchronization by circuit implementation," Nonlinear Dynamics, vol. 68, no. 1-2, pp. 137-149, 2012.
[29] Z. Ruo-Xun and Y. Shi-Ping, "Chaos in fractional-order generalized lorenz system and its synchronization circuit simulation," Chinese Physics B, vol. 18, no. 8, pp. 3295-3302, 2009.

[30] C. K. Ahn, S. Jung, S. Kang, and S. Joo, "Adaptive $\mathrm{H}_{\infty}$ synchronization for uncertain chaotic systems with external disturbance," Communications in Nonlinear Science and Numerical Simulation, vol. 15, no. 8, pp. 2168-2177, 2010.

[31] C. K. Ahn, "Adaptive $\mathrm{H}_{\infty}$ anti-synchronization for time-delayed chaotic neural networks," Progress of Theoretical Physics, vol. 122, no. 6, pp. 1391-1403, 2009.

[32] C. K. Ahn, "T-S fuzzy Ho synchronization for chaotic systems via delayed output feedback control," Nonlinear Dynamics, vol. 59, no. 4, pp. 535-543, 2010.

[33] C. K. Ahn, "Output feedback $\mathrm{H}_{\infty}$ synchronization for delayed chaotic neural networks," Nonlinear Dynamics, vol. 59, no. 1-2, pp. 319-327, 2010.

[34] C. K. Ahn, "An $\mathrm{H}_{\infty}$ approach to anti-synchronization for chaotic systems," Physics Letters A: General, Atomic and Solid State Physics, vol. 373, no. 20, pp. 1729-1733, 2009. 


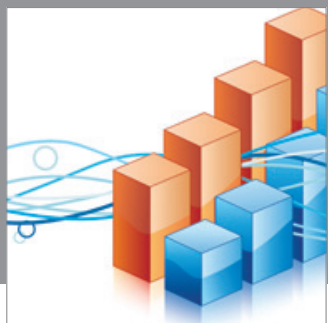

Advances in

Operations Research

mansans

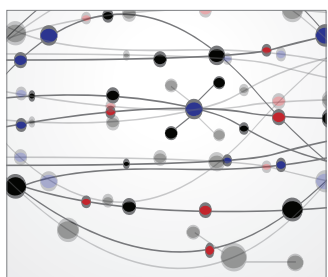

The Scientific World Journal
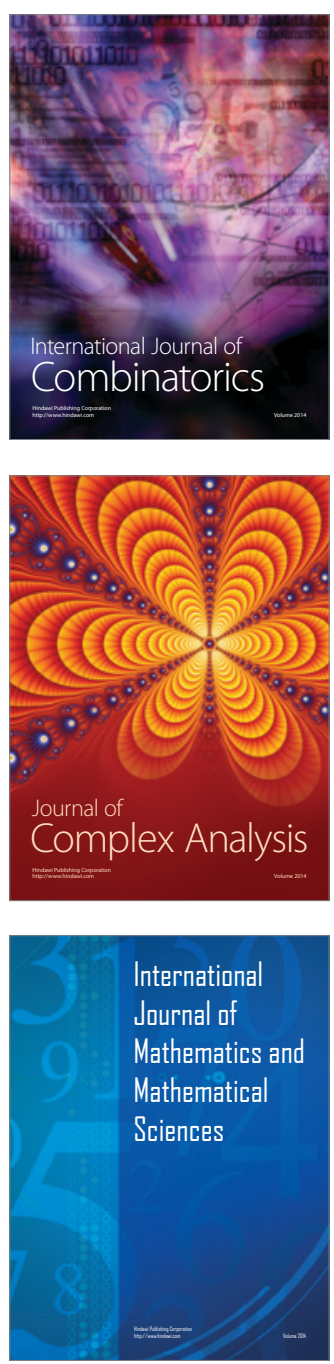
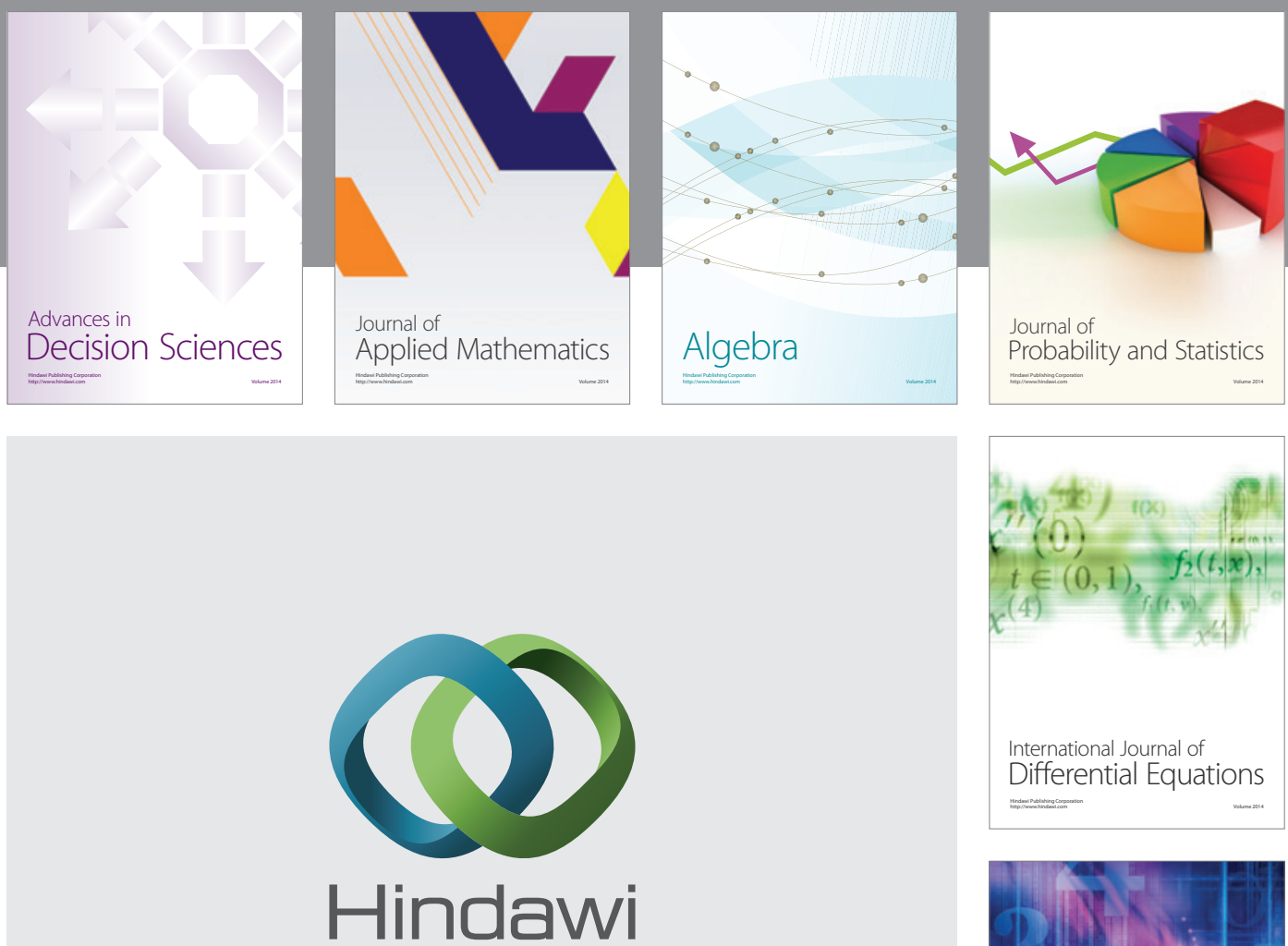

Submit your manuscripts at http://www.hindawi.com
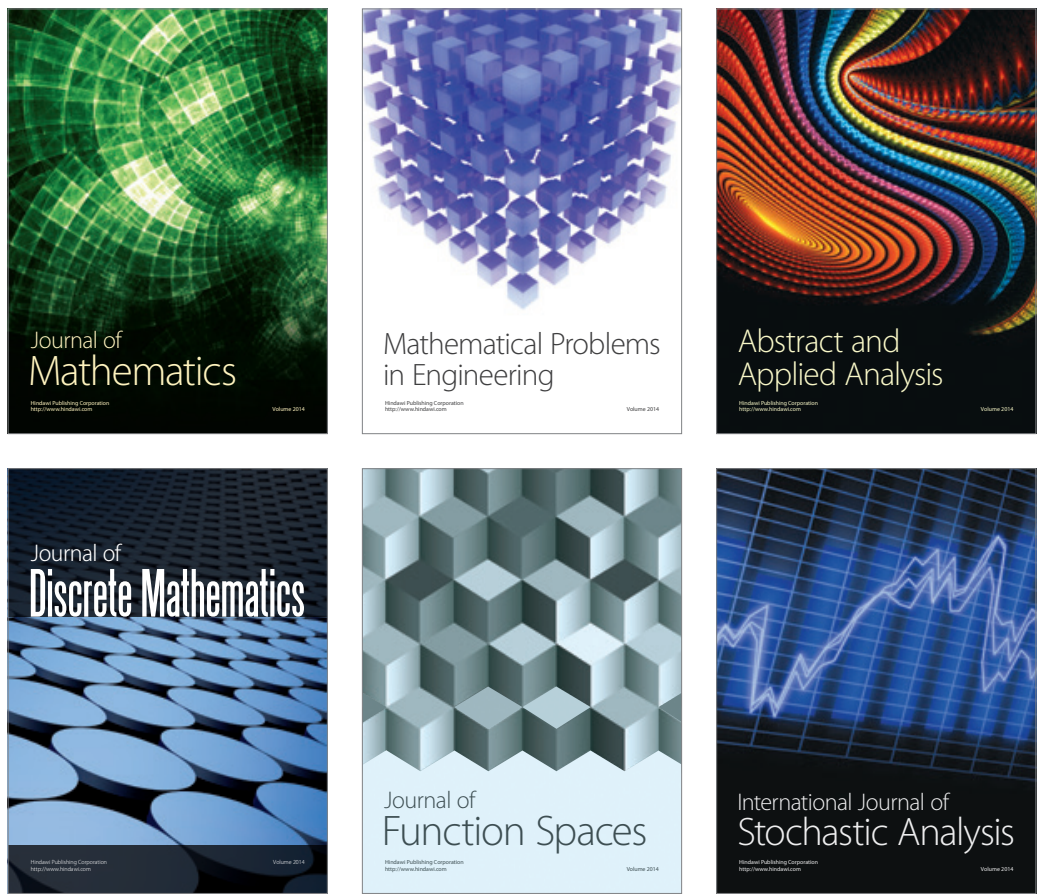

Journal of

Function Spaces

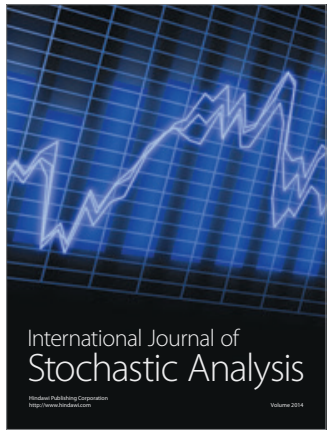

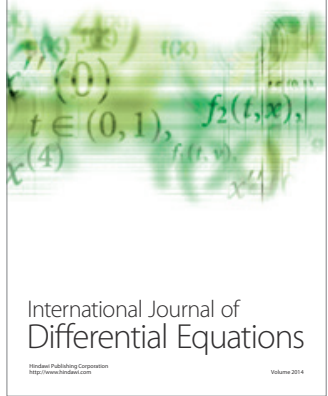
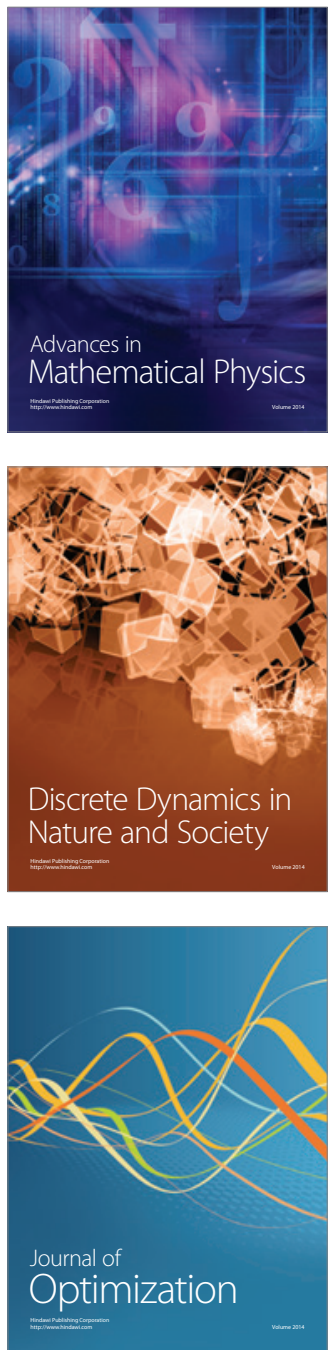